\section{A E E T}

ASOCIACIÓN ESPAÑOLA DE ECOLOGÍA TERRESTRE

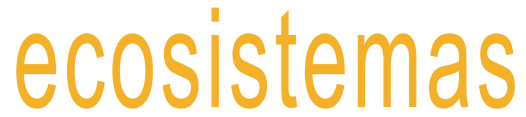

REVISTA CIENTÍFICA DE ECOLOGÍA Y MEDIO AMBIENTE

ISSN 1697-2473 / Open access

disponible en www.revistaecosistemas.net

\title{
Vulnerabilidad y resiliencia de ecosistemas forestales frente a episodios extremos de sequía
}

\author{
F. Lloret ${ }^{1,2, *}$ \\ (1) CREAF, Cerdanyola del Vallès 08193, España \\ (2) Univ Autònoma Barcelona, Cerdanyola del Vallès 08193, España
}

* Autor de correspondencia: F. Lloret [francisco.lloret@uab.cat]

> Recibido el 13 de octubre de 2012, aceptado el 12 de noviembre de 2012.

\begin{abstract}
Lloret, F. (2012). Vulnerabilidad y resiliencia de ecosistemas forestales frente a episodios extremos de sequía. Ecosistemas 21(3):85-90. Doi.:10.7818/ECOS.2012.21-3.11

En los últimos años se están observando fenómenos de decaimiento que afectan a diferentes tipos de bosque y matorral a una escala global, y que con frecuencia se asocian a anomalías climáticas de sequía. El fenómeno presenta patrones espaciales evidentes y no es homogéneo en todas las localidades con características climáticas similares. Esto indica la existencia de diferentes factores que contribuyen al decaimiento -como las características del suelo relacionadas con la disponibilidad de agua- y de una apreciable capacidad de resiliencia de estos ecosistemas. De hecho, para que se produzca un declive poblacional debe existir un desequilibrio entre mortalidad y reclutamiento: cualquier factor que disminuya la mortalidad o favorezca el reclutamiento tenderá a potenciar esta resiliencia. Por tanto, los cambios en la composición de la comunidad y la eventual sustitución de especies se producirían cuando el resultado de este balance sea diferente entre las especies. Además, los efectos de estos episodios extremos de sequía pueden verse acentuados por la confluencia con otras perturbaciones, particularmente con aquellas que tienden a aumentar en las condiciones asociadas a la anomalía climática. Este es el caso de los incendios, con una especial incidencia en los ecosistemas mediterráneos.

Los episodios de decaimiento deberían ayudar a incorporar el contexto de cambio climático a la gestión forestal. En particular sería conveniente introducir una aproximación proactiva que favorezca los mecanismos que tienden a incrementar la resiliencia de los ecosistemas terrestres y su adaptación a las nuevas condiciones climáticas, como por ejemplo la selección de especies y genotipos, la adecuación de la estructura del bosque a nivel de rodal a las condiciones climáticas futuras y la distribución de estos elementos a escala de paisaje.
\end{abstract}

Palabras clave: cambio climático, sequía, episodios climáticos extremos, decaimiento forestal, gestión forestal, resiliencia

Lloret, F. (2012). Vulnerability and resilience of forest ecosystems to extreme drought episodes. Ecosistemas 21(3):85-90. Doi.: 10.7818/ECOS.2012.21-3.11

Drought-induced forest die-off has been recently reported in many locations abroad, and also in some shrublands. The phenomena show recognizable spatial patterns and it is not homogenous in localities with similar climatic characteristics. Therefore, we can identify different drivers contributing to die-off, for instance soil properties related soil availability, but also the existence of a remarkable resilience in these ecosystems. In fact, population decline would occur as a result of the unbalance between mortality and recruitment. Any factor contributing to diminish mortality or favouring recruitment would enhance resilience, and shifts in community composition would occur when the balance between mortality and recruitment will be different across species. Also, the impacts of these events would be intensified by the synergy with other disturbances, such as wildfires in Mediterraneantype ecosystems, particularly if these disturbance generate conditions similar to those resulting from the climatic anomaly.

The die-off events can help to incorporate climate change context to forest management. Particularly, a pro-active approach by favouring the mechanisms contributing to forest resilience would be desirable. These actions include species and genotypes selection, forest structure adequate to optimize resource use in the oncoming climatic conditions, and distribution of these elements at landscape level.

Key words: climate change, drought, extreme climatic episode, forest die-off, forest management, resilience

\section{La tendencia climática}

El aumento de las temperaturas es indudablemente la señal más universal del cambio climático. En el caso de los ecosistemas de la cuenca mediterránea, la tendencia de las precipitaciones no es tan clara. En cualquier caso, no se ha detectado un gran incremento de las precipitaciones en las últimas décadas, ni se espera que aumenten en el futuro. Por tanto, nos enfrentamos a una situación de un aumento de la aridez. Además, los estudios más recientes están fijando nuestra atención en un componente del cambio climático que en un principio había pasado bastante desapercibido. Se trata del incremento de la variabilidad climática que afectaría a temperatura y precipitación. Intuitivamente, no es difícil aceptar que si un sistema tan complejo como el climático se está viendo alterado de una forma rápida, el ajuste a un nuevo estado no es inmediato y presenta fuertes oscilaciones hasta que las diferentes retroacciones implicadas ajustan sus mutuas influencias. El incremento de la variabilidad implica que aumenta la probabilidad de tener una situación climática que se consideraba extrema en las condiciones anteriores. Este hecho se aprecia bien en los gráficos que representan la tendencia climática a escala anual, por ejemplo de temperaturas medias (Fig. 1). Aunque la tendencia en promedio sea un aumento, los dientes de sierra nos indican esa variabilidad y los datos disponibles indican que la magnitud de esos dientes de 


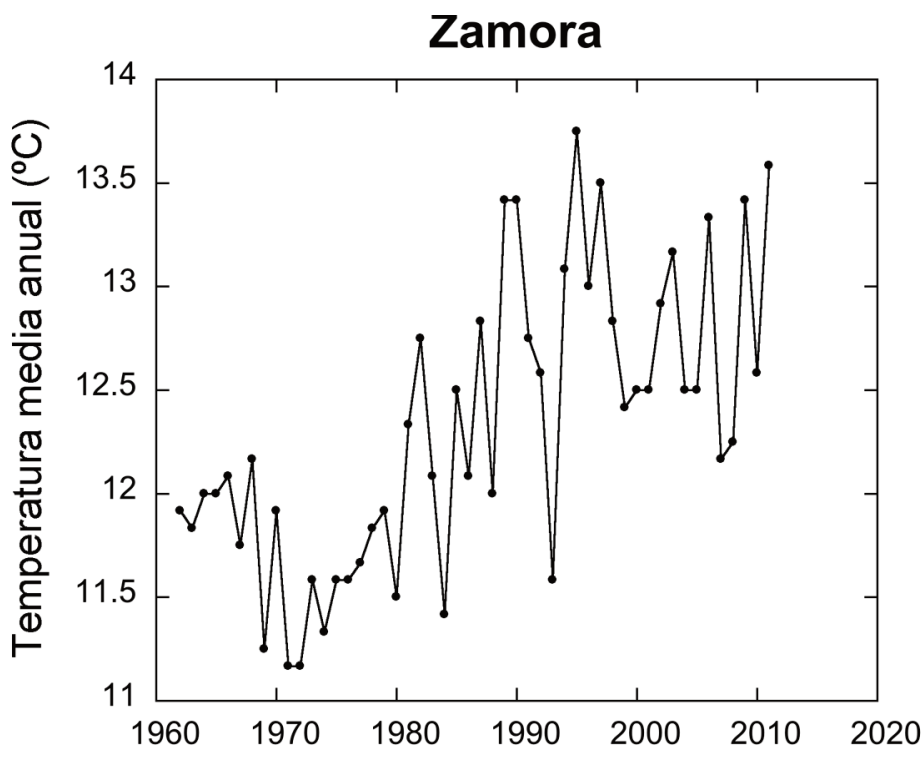

Figura 1. Serie climática de las temperaturas medias anuales de Zamora (España), mostrando la tendencia promedio al aumento de las temperaturas y la tendencia al aumento de la variabilidad, que resulta en la aparición de episodios climático extremos. Datos procedentes de la Agencia Estatal de Meteorología, Gobierno de España.

sierra tiende a aumentar. Los episodios climáticos extremos se corresponderían con los dientes de sierra, y aunque su definición puede estar sujeta a cierta apreciación subjetiva, existen técnicas estadísticas que ayudan a su determinación. Es particularmente importante comprender que estos episodios extremos pueden implicar que se sobrepasen umbrales de tolerancia ecológica no reversibles. El caso más obvio y extremo a nivel individual es la muerte: un organismo que muere como consecuencia de esas condiciones extremas no retorna a la vida cuando las condiciones vuelven a ser aptas para su supervivencia. Si sobrevive, la capacidad de retornar al estado previo al episodio corresponde a lo que los ecólogos llamamos resiliencia. Hay que recordar, sin embargo, que este término no tiene siempre esta acepción y en otros ámbitos se aplica para describir la capacidad de permanecer inalterado ante una modificación del medio, propiedad que los ecólogos solemos denominar como resistencia. Por tanto, podemos reconocer que el cambio climático implica tendencias promedio, cuyas consecuencias se reflejarían a escalas temporales relativamente amplias (por ejemplo, décadas), y tendencias de la variabilidad que pueden tener efectos a escalas más pequeñas (por ejemplo, años, si consideramos como unidad temporal el ciclo anual de fluctuaciones estacionales) (Easterling 2000). En principio, las tendencias promedio podrían ser adecuadas para los modelos que analizan cambios futuros en patrones que integran procesos que duran varios años, como la distribución regional de las especies o los biomas.

Por tanto es pertinente analizar la importancia efectiva de estos episodios climáticos extremos, ya que potencialmente pueden afectar al ajuste de las comunidades al nuevo escenario climático. La situación más obvia se da cuando el episodio extremo tiene el mismo sentido que la tendencia general. Entonces cabe esperar que el episodio precipite el resultado de esta tendencia general. Sin embargo, la situación opuesta, mucho menos considerada en la literatura, en la que el episodio se contrapone a la tendencia general puede retardar sus efectos, por ejemplo favoreciendo el reclutamiento de las poblaciones (Matías et al 2012). Además, hay que considerar los efectos de estos episodios en su contexto temporal específico, es decir en la secuencia de condiciones que se han producido anteriormente. De hecho, el aumento de la variabilidad climática implicaría no únicamente una mayor intensidad (mayor distancia entre las puntas de los dientes de sierra que mencionábamos antes) sino también una frecuencia más elevada de los episodios extremos (Della-Marta et al. 2007), y este aumento de su recurrencia puede potenciar su efecto.

\section{Defoliaciones masivas y sequía en la Península Ibérica}

En el caso de la cuenca mediterránea, los episodios de sequía cobran particular importancia. Los datos de los que disponemos confirman la tendencia a un aumento de estos episodios (DellaMarta et al. 2007) y los modelos climáticos apuntan a que esta tendencia continuará (Sánchez et al. 2011). Algunos casos de decaimiento del bosque, como los analizados para el pino albar (Martínez-Vilalta et al. 2012, Sánchez-Salguero et al. 2012), el pino laricio (Sánchez-Salguero et al. 2012), o el alcornoque (FernándezCancio et al. 2012) en este mismo número de Ecosistemas, estarían, al menos en parte, relacionados con estos episodios y formarían parte de un fenómeno global (Allen et al. 2010). Además de los mecanismos implicados en este proceso de decaimiento (Fernández-Cancio et al. 2012; Martínez-Vilalta et al. 2012; Sánchez-Salguero et al. 2012), ¿qué sabemos de sus características? Aunque no existen estudios exhaustivos comparativos que permitan analizar con precisión los patrones del fenómeno, una primera apreciación indica que:

- la vegetación afectada por defoliaciones intensas que podemos relacionar con episodios de sequía no se limita a los bosques, sino que también se han observado procesos de decaimiento en matorrales mediterráneos (del Cacho y Lloret 2012) (Fig. 2).

- las zonas afectadas suelen presentar patrones espaciales complejos, a menudo difusos, debido al diferente grado de afectación de la masa forestal y a la propia idiosincrasia de las especies (Carnicer et al. 2011). No suelen observarse frentes de defoliación bien definidos y continuos, o patrones de progreso concéntrico, como en algunas plagas forestales. A diferencia de otras regiones, como el suroeste de Norteamérica, donde el decaimiento afecta a enormes extensiones, en nuestro entorno el área de los rodales afectados suele ser limitada, probablemente debido a la propia heterogeneidad del bosque y del medio.

- el medio edáfico juega un papel fundamental en los patrones espaciales de decaimiento. En un estudio realizado en encinares de las montañas de la costa catalana, Lloret et al. (2004) mostraron variaciones de la defoliación que iban desde el 90 al $45 \%$ en pocos metros de distancia, los cuales correspondían a la transición entre conglomerados masivos altamente infranqueables a las raíces y esquistos fisurados donde las raíces podían a acceder a reservas de agua más profunda. La profundidad del suelo y su capacidad de retención del agua suelen aparecer como unas de las variables explicativas del decaimiento más consistentes (Galiano et al. 2010; 2012; Candel-Pérez et al. 2012; Linares et al. 2012).

- la densidad de árboles o el área basal de los rodales, suele corresponder con mayores niveles de afectación que puede interpretarse como resultado de una mayor competencia (Galiano et al 2010; 2012; Linares et al. 2010a; b; Candel-Pérez et al. 2012).

- el decaimiento no siempre se produce de forma abrupta. Aunque se identifican situaciones de defoliación masiva en intervalos cortos de tiempo, que en nuestro caso corresponden con episodios de sequía, con frecuencia el decaimiento implica un proceso crónico que se prolonga en el tiempo (Carnicer et al. 2011; Camarero et al. 2011; Hereş et al. 2012).

- en algunas especies la defoliación es reversible. Es el caso de las encinas en las que se ha documentado que la mayoría de ellas pueden rebrotar de la cepa o incluso de la copa (Lloret et al. 2004; Galiano et al. 2012). Existen evidencias de que los efectos del episodio a nivel fisiológico persisten varios años (Peñuelas et al. 2000) y de que la recurrencia de los eventos promueve la muerte final del árbol (Lloret et al. 2004). Los mecanismos implicados en esta secuencia de acontecimientos se discute con detalle en Martinez-Vilalta et al. (2012) para el caso del pino albar y en gran medida puede ser extrapolable a otras especies.

- la historia de los bosques, y en particular el tipo de manejo realizado en ellos puede influir en la vulnerabilidad de los bosques a los episodios de sequía. De hecho, el estudio de los datos propor- 

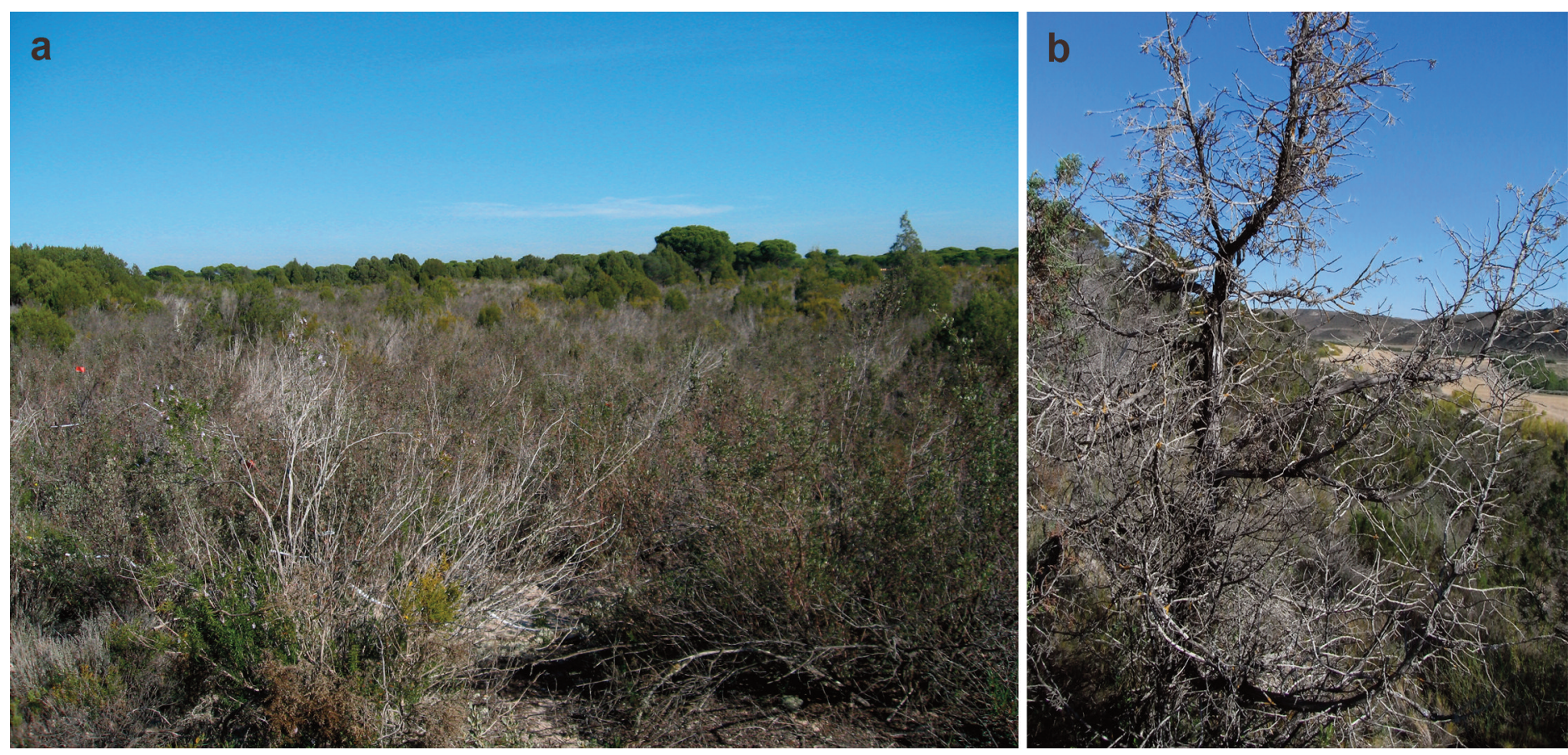

Figura 2. Formaciones arbustivas afectadas por decaimiento y mortalidad. a: monte blanco afectado por un episodio de sequía en el año hidrológico 2004-2005, Parque Nacional de Doñana (Fecha de la fotografía: 2008). b: Sabinar de Juniperus phoenicea, Monegros (Fecha de la fotografía: 2012). Autor de las fotografías: Francisco Lloret.

cionados por la red española de inventarios forestales (IFN) muestra que las características estructurales de los bosques, que están relacionadas directamente con el manejo, pueden tener más importancia en la mortalidad y el crecimiento que las variables climáticas (Vilà-Cabrera et al. 2011). Así mismo, Camarero et al. (2011) han estudiado los anillos de crecimiento de abetos de los Pirineos y han comprobado como aquellas poblaciones con mayor decaimiento habían experimentado históricamente mayores pulsos de crecimiento relacionados con niveles altos de explotación del bosque.

- el decaimiento es un fenómeno multifactorial en el que intervienen diferentes agentes. Además de los mencionados (variables topoclimáticas y edáficas, manejo, estructura del rodal), cabe destacar el papel de patógenos, plagas e incluso plantas hemiparásitas como el muérdago (Galiano et al. 2010; 2012; Candel-Pérez et al. 2012, Sangüesa-Barreda et al. 2012). A menudo no es fácil distinguir cual es la causa última del decaimiento y eventual muerte, ya que árboles infectados serían más vulnerables a la sequía (Linares et al. 2010b; Martínez-Vilalta et al. 2012), mientras que el estrés inducido por la sequía podría facilitar la infección. Sin duda hace falta una mayor colaboración entre equipos de fitopatólogos y ecofisiólogos para poder discriminar mejor la contribución de estos agentes en el fenómeno del decaimiento.

- el decaimiento puede considerarse un tipo particular de perturbación. Sin embargo, en ocasiones su aparición y desarrollo se produce de forma continuada en el tiempo, como se ha comentado anteriormente. Además, la alteración del medio presenta ciertas diferencias con respecto a otras perturbaciones frecuentes en la región, ya que los árboles se suelen mantener en pie durante bastantes años, generándose unos gradientes de radiación y mantillo más graduales que en el caso de otras perturbaciones. Así, los incendios implican transformaciones más intensas del suelo que en el caso del decaimiento, ya que la combustión produce en primera instancia una mayor fertilización del suelo, pero la erosión y la lixiviación puede incrementarse a medio plazo. En las perturbaciones causadas por el viento o la nieve, la caída de troncos, ramas y hojas se produce de repente, abriéndose claros bien definidos en el dosel. Si la perturbación se debe a la explotación forestal, la modificación del dosel, del suelo y de la distribución de los restos vegetales también suele ser más intensa y los gradientes abióticos inducidos son más abruptos que en el caso del decaimiento. No obstante, faltan estudios que cuantifiquen los cambios en las características del medio y su repercusión en el funcionamiento del bosque y en su eventual regeneración.

\section{Resiliencia de los ecosistemas forestales}

A pesar del gran interés reciente en el decaimiento forestal y en los procesos asociados a la mortalidad de los árboles, los estudios dedicados a abordar las consecuencias de los episodios extremos de sequía en la dinámica de los bosques son todavía escasos. Una primera hipótesis que se plantea es que estos fenómenos promueven cambios rápidos en las comunidades a través de la muerte de las especies dominantes y su substitución por otras especies más adaptadas a las nuevas condiciones climáticas. Sin embargo, este modelo parece demasiado sencillo. Teniendo en cuenta que los datos son aún escasos, parece que en muchos casos las situaciones climáticas extremas no comportan un decaimiento, al menos que sea perceptible de forma inmediata. De hecho, el número de estudios que documentan transformaciones de la vegetación inducidas por episodios de sequía es sorprendentemente escaso, aunque esto puede deberse en parte a la falta de prospecciones a largo plazo. También puede deberse a que los cambios observados en la vegetación podrían tener un carácter transitorio. Esta afirmación es extensiva a los bosques de la cuenca mediterránea. Por ejemplo, es conocida la gran capacidad de las encinas para recuperar su dosel verde después de defoliaciones masivas (Lloret et al. 2004; Galiano et al. 2012) y ello permite que las defoliaciones observadas en encinares puedan revertir después de un tiempo. Estas observaciones sugieren la existencia de procesos estabilizadores en las poblaciones vegetales, es decir, que los bosques disponen de mecanismos de resistencia y de resiliencia frente a estos episodios y que cualquier acción que los promueva ayudará a minimizar los efectos de los mismos (Lloret et al. 2012).

Un marco conceptual sencillo para analizar estos cambios en la comunidad es entender la continuidad de la población de una determinada especie como un balance a largo plazo de su mortalidad y su reclutamiento, y entender la continuidad de la comunidad como el mantenimiento de las abundancias relativas de las diferentes especies, particularmente de las dominantes. Este es un plan- 
teamiento simplificado ya que, por ejemplo, existen técnicas bien conocidas capaces de modelizar la tendencia demográfica a partir de los valores de estos parámetros en las diferentes clases de edad de las poblaciones. Teóricamente, los eventos de decaimiento masivo pueden conducir a cambios en la composición de bosques mixtos de forma inmediata o a medio plazo en las situaciones en que haya: 1) mortalidad diferencial de los adultos o del regenerado de las especies implicadas; 2) reclutamiento diferencial de las especies debido a los cambios microambientales inducidos por el episodio; o 3) reclutamiento diferencial de las especies debido a los filtros bióticos resultantes de la sequía. Por tanto, a priori, cualquier mecanismo que opere desde el nivel genético y su expresión fenotípica al de comunidad minimizando estas situaciones actuará como elemento estabilizador. El análisis de los procesos resultantes y su modelización nos permitirá conocer los umbrales de resistencia de las comunidades forestales y su capacidad de resiliencia ante estos eventos.

Entre los procesos a nivel de comunidad que pueden reforzar esta inercia al cambio podemos mencionar las interacciones bióticas que promuevan la supervivencia, y el reclutamiento. Es el caso de 1) la facilitación y el mutualismo, que han sido bien estudiados en sistemas mediterráneos, particularmente en las fases de establecimiento, 2) de las eventuales disminuciones de la competencia que se puedan producir por reducciones de la densidad de las poblaciones de las especies vegetales dominantes, o 3) de la reducción de la intensidad de las relaciones antagonistas con patógenos o fitófagos que también se vean afectados por la sequía (Lloret et al 2012). También hay que destacar las relaciones de complementariedad de nicho y redundancia funcional entre especies vegetales. Esta relación de complementariedad y redundancia explicaría el efecto amortiguador que tiene la diversidad vegetal en la respuesta de algunas propiedades funcionales del ecosistema frente a los episodios de sequía (Díaz-Delgado et al. 2002). Así mismo, nos queda mucho por averiguar sobre el papel de los procesos edáficos controlados por las comunidades microbianas y su repercusión en la dinámica de las comunidades vegetales, aunque empezamos a tener indicios de que estas comunidades microbianas responden también de forma rápida y significativa a los eventos de decaimiento (Curiel-Yuste et al., en prensa).

Finalmente, hay que considerar que las consecuencias de los episodios de sequía no se pueden desligar de otras perturbaciones, algunas de las cuales pueden actuar de forma sinérgica. Entre ellas podemos destacar los incendios y las plagas forestales. La tendencia climática y el cambio de los usos del territorio han provocado en las últimas décadas que los incendios forestales se hayan convertido en una de las principales problemáticas de los bosques de la cuenca mediterránea. Aunque los incendios están claramente determinados por las condiciones climáticas, las cuales se han hecho más propicias para ellos, su dependencia de la existencia de combustible hace que su regulación sea más compleja (Lloret et al. 2009). En principio, las condiciones climáticas que aumentan el riesgo de los incendios forestales y de eventuales defoliaciones masivas y decaimiento son coincidentes. Por tanto, estas dos situaciones tendrían efectos sinérgicos con un gran potencial para promover cambios bruscos en los ecosistemas forestales, ya que la regeneración se vería limitada por la propia tendencia climática a la aridez. Una situación particularmente preocupante se daría en zonas climáticas, como las de montaña, vulnerables al decaimiento y con comunidades constituidas por especies poco resilientes al fuego, ya que históricamente han sido poco afectadas por esta perturbación. Sin embargo, a más largo plazo, esa misma aridez implicaría una reducción del combustible y una limitación de los incendios. De hecho algunos modelos globales de vegetación proyectan situaciones futuras de disminución de los incendios forestales debido a una reducción del combustible, a pesar del incremento del riesgo climático de incendios (Kloster et al. 2012). A su vez, el cambio climático está modificando profundamente el impacto de las plagas forestales en bosques de coníferas boreales y de regiones templadas, al modificar el ciclo biológico y la distri- bución de los patógenos o insectos implicados, a la vez que aumenta la vulnerabilidad de los vegetales (Ayres y Lombardero 2000). Aunque en general han sido menos consideradas en bosques mediterráneos hasta ahora, existen indicios de que estas interacciones antagonistas pueden tener un papel muy relevante en el decaimiento (Martínez-Vilalta et al. 2012), y sin duda deben ser exploradas en más profundidad.

\section{Consecuencias para la gestión}

La situación en la que nos encontramos de cambio climático implica nuevos retos a la gestión de los ecosistemas terrestres, y en concreto de los forestales. El nuevo escenario afecta a todos los sistemas ecológicos en mayor o menor grado, de forma que cualquier actuación concreta de manejo debe considerar que el medio físico está cambiando de forma rápida y en una dirección que conocemos a grandes rasgos. La gestión de los bosques es especialmente sensible a esta situación por cuanto las actuaciones presentes tienen repercusión décadas después, cuando sabemos que las condiciones climáticas, un parámetro fundamental en su funcionamiento, serán muy diferentes. A veces se contraargumenta afirmando nuestro desconocimiento exacto del futuro, y prevalece la opinión de que ante esta incertidumbre es mejor actuar a partir de la experiencia pasada. Sin embargo, en términos generales podríamos prescindir de esa incertidumbre, por cuanto el cambio ya se está produciendo, lo estamos detectando y es de gran magnitud. Por tanto, a la hora de establecer las pautas de manejo y planificación forestal en la cuenca mediterránea, es menos incierto asumir una mayor aridez futura que unas condiciones climáticas como las actuales o las pasadas. Conseguir que este posicionamiento se establezca firmemente en el ámbito de la gestión es el primer reto al que nos enfrentamos, y que se resume en la premisa "el bosque responderá a cualquier actuación de manejo forestal que se realice en el presente en unas condiciones climáticas más áridas que las actuales".

Además, si como hemos visto, esta tendencia climática está puntuada por situaciones extremas y poco predecibles, el manejo debe también desarrollar herramientas para enfrentarse a ellas. Ante la situación de certeza a medio plazo y de incertidumbre a corto plazo, una estrategia apropiada sería combinar la planificación a largo plazo con la capacidad de intervención rápida, reconociendo que no hay una única solución a todas las situaciones que se dan (Spittlehouse y Steward 2003; Hobbs et al. 2006; Millar et al. 2007). La planificación a medio plazo implica ampliar los periodos en los cuales se aplican y se evalúan los planes de actuación. De esta forma se podría promediar los valores medios anuales (por ejemplo de crecimiento, producción, mortalidad) de un número mayor de años para tener una idea más fidedigna del estado de esas variables. Es de sobra conocido que en una serie de datos estadísticos, cuando la variabilidad aumenta se necesita un mayor número de casos para mantener la misma fiabilidad de la estima de la media. La capacidad de intervención a corto plazo en una situación de elevado estrés climático se puede conseguir con una elevada flexibilidad en la asignación de recursos, que implica aceptación de riesgos, capacidad de reevaluar las condiciones con frecuencia y la voluntad de modificar los planes de ejecución previstos (Hobbs et al. 2006). La alternativa de mantener inmovilizados los recursos a la espera de que se produzca esa situación parece poco viable en un escenario como el actual de gran escasez de recursos económicos. Sin embargo, esta estrategia se encuentra con el reto de romper la inercia a mantener los actuales periodos de asignación y ejecución de actividades, sujetos a los ciclos presupuestarios anuales y a los eventos electorales.

Dada la magnitud de la transformación del medio a medio y largo plazo del cambio climático y la naturaleza estocástica de los episodios climáticos extremos, las actuaciones encaminadas a aumentar la resistencia de los bosques en situaciones concretas de sequía ponen a prueba la capacidad de intervención rápida. Estos elementos implican una priorización de las zonas de actuación, en favor de bosques con valores destacados (Millar et al. 2007). 
Una aproximación preventiva frente al resultado poco deseable de los episodios extremos de sequía es aumentar la resiliencia de los ecosistemas forestales (Spittlehouse y Steward 2003). Esta estrategia se basa en gestionar procesos ecológicos y es particularmente adecuada cuando nos encontramos frente a fluctuaciones del medio de manera que es previsible que las condiciones de estrés disminuyan o aumenten con el tiempo, aunque sea de forma transitoria. Además, en este caso se tiene la ventaja de aplicar actuaciones similares a las que se plantean en el escenario conocido de aumento generalizado de la aridez. De hecho, el dramatismo asociado a los episodios extremos de sequía, con defoliaciones masivas y mortalidades apreciables, puede favorecer la toma de conciencia de la tendencia climática a la aridez y por tanto facilitar estas actuaciones. De esta forma se conseguiría superar uno de los grandes inconvenientes de la gestión del cambio climático: la dificultad de trasladar el escenario futuro a la realidad actual.

Sin embargo, ante un escenario de cambio climático inexorable, en muchos casos el incremento de la resiliencia no será suficiente y es previsible la transformación de muchos ecosistemas forestales. Ante esta situación, la alternativa sería acompañar la transformación gradual del sistema y favorecer su adaptación a las nuevas condiciones, con el objetivo de minimizar los efectos poco deseables de los cambios catastróficos (Millar et al. 2007). Una ventaja de esta estrategia, compartida con el potenciamiento de la resiliencia, es que tenemos los conocimientos suficientes para establecer algunos principios generales y la capacidad de desarrollar líneas de investigación acordes con estas perspectivas. El hecho de que la tendencia climática tenga el mismo sentido de aridez que los episodios de sequía hace que en muchos casos las actuaciones entre estas estrategias sean coincidentes. Por ejemplo, se puede 1) promover determinadas especies o genotipos menos vulnerables a la sequía adecuando su localización a los nuevos escenarios, 2) potenciar una diversidad que facilite la redundancia funcional ante un rango amplio de condiciones climáticas, 3) modificar la estructura del bosque para minimizar los efectos negativos de la competencia cuando los recursos hídricos son escasos, 4) permitir la coexistencia de diferentes clases de edad a escala de rodal o de estados sucesionales a nivel de paisaje, y 5) favorecer los procesos que mejoran la regeneración y el reclutamiento de nuevos individuos, como por ejemplo determinadas interacciones bióticas o estructuras de paisaje que favorecen la dispersión.

Cuando las medidas preventivas no han sido suficientes, y los episodios climáticos resultan en fuertes impactos en las comunidades forestales llevando a estados no deseados, también disponemos de un importante conocimiento en técnicas de restauración que se aplican habitualmente en situaciones posteriores a las perturbaciones. Sin entrar en detalles, un elemento clave en estas actuaciones es la potenciación del regenerado, siendo conscientes de que la mayor frecuencia de sequías extremas o el aumento gradual de la aridez pondrá en riesgo su viabilidad futura. Sin duda, estas actuaciones paliativas necesitan todavía de un importante bagaje de conocimiento que responda a preguntas de carácter práctico específicas para los episodios de sequía, como por ejemplo la conveniencia de cortar determinados árboles con síntomas de decaimiento atendiendo a sus probabilidades de supervivencia o crecimiento posterior.

Los problemas planteados al manejo de los bosques por los episodios de sequía constituyen un caso evidente en el que la gestión adaptativa es particularmente apropiada dada la estocasticidad e incertidumbre asociada a estos fenómenos. Aunque no sabemos cuándo se van repetir, sabemos que aparecerán y que hay localidades y características de los bosques que los hacen más vulnerables. También conocemos algunos de los principios ecológicos que gobiernan la respuesta de los ecosistemas a estas situaciones, disponemos de las herramientas para modelizarlas y seguiremos aprendiendo más conforme contrastemos la efectividad de las actuaciones realizadas. Sin embargo, a pesar de nuestros esfuerzos por minimizar su impacto, no podemos evitar la inercia climática al calentamiento y la aridez, la cual previsiblemente conllevará una pérdida importante de superficie forestal y su substitución por formaciones menos estructuradas como matorrales y pastos. Por tanto, además de promover la adaptación de los bosques a la nueva situación climática, a medio y largo plazo no tenemos más alternativa que adaptar la visión que nuestra sociedad tiene de estas formaciones menos estructuradas y ponerlas en valor por lo que tienen de crisol de biodiversidad y de proveedoras de servicios fundamentales, como la regulación de las cuencas hidrográficas o la conservación de los suelos.

\section{Agradecimientos}

Este pequeño escrito sólo tiene sentido desde el esfuerzo de muchos colegas, algunos de cuyos trabajos no han podido quedar reseñados: sirvan estas palabras de disculpas y agradecimiento hacia ellos. Quisiera agradecer especialmente las informaciones y opiniones intercambiadas desde hace ya tiempo con los miembros de GLOBIMED y en particular con J. Martínez-Vilalta, L. Galiano, F. Valladares, A. Escudero, J.M. Olano y J.J. Camarero. Los estudios realizados por el autor han sido posibles gracias a dos ayudas del ICTS-RBD, de las ayuda AGAUR 2009 SGR 00247 (Generalitat de Catalunya),y de los proyectos CGL 200601293/BOS, CGL 2009-08101 and BOS Consolider-Ingenio Montes CSD 2008-0040.

\section{Referencias}

Allen, C.D., Macalady, A.K., Chenchouni, H., Bachelet, D., McDowell, N., Vennetier, M., Kitzberger, T., Rigling, A., Breshears, D.D., Hogg, E.H., et al. 2010. A global overview of drought and heat-induced tree mortality reveals emerging climate change risks for forests. Forest Ecology and Management 259:660-684.

Ayres, M.P., Lombardero, M.J. 2000. Assessing the consequences of global change for forest disturbance from herbivores and pathogens. Science of the Total Environment 262:263-286.

Camarero, J.J., Bigler, C., Linares, J.C., Gil-Pelegrín, E. 2011. Synergistic effects of past historical logging and drought on the decline of Pyrenean silver fir forests. Forest Ecology and Management 262:659-669.

Candel-Pérez, D., Linares J.C., Viñegla, B., Lucas-Borja, M.E. 2012. Assessing climate-growth relationships under contrasting stands of co-occurring Iberian pines along an altitudinal gradient. Forest Ecology and Management 274:48-57.

Carnicer, J., Coll, M., Ninyerola, M., Pons, X. Sánchez, G., Peñuelas, J. 2011. Widespread crown condition decline, food web disruption, and amplified tree mortality with increased climate change-type drought. Proceedings National Academy of Sciences 108: 1474-1478.

Curiel Yuste, J., Barba, J., Fernandez-Gonzalez, J., Fernandez-Lopez, M., Mattana, S., Martinez-Vilalta, J., Nolis, P., Lloret, F. 2012, en prensa. Changes in soil bacterial community triggered by drought-induced gap succession preceded changes in soil $\mathrm{C}$ stocks and quality. Ecology and Evolution. DOI: 10.1002/ece3.409.

Del Cacho, M., Lloret, F. 2012. Resilience of Mediterranean shrubland to severe drougth episode: the role of seed bank and seedling establishment. Plant Biology 14:458-466.

Díaz-Delgado, R., Lloret, F., Pons, X., Terradas, J. 2002. Satellite evidence of decreasing resilience in Mediterranean plant communities after recurrent wildfires. Ecology 83:2293-2303.

Della-Marta P., Haylock, M.R., Luterbacher, J., Wanner, H. 2007. Doubled length of western European summer heat waves since 1880. Journal of Geophysical Research 112:D15103.

Easterling, D.R. 2000. Climate Extremes: Observations, Modeling, and Impacts Science 289:2068-2074.

Fernández-Cancio, A., Sánchez-Salguero, R., Navarro-Cerrillo, R.M., Manrique Menéndez, E., Fernández Fernández, R., Gil, P.M. 2012. Efectos del cambio climático sobre el decaimiento de los alcornocales españoles. Una aproximación fitoclimática para la futura gestión. Ecosistemas 21(3):50-62.

Galiano, L., Martínez-Vilalta, J., Lloret, F. 2010. Drought-induced decline of Scots pine stands in Central Pyrenees is mediated by multiple predisposing factors. Ecosystems 13:978-991.

Galiano, L., Martínez-Vilalta, J., Sabaté, S., Lloret, F. 2012. Determinants of drought effects on crown condition and their relationship with depletion of carbon reserves in a Mediterranean holm oak forest. Tree Physiologist, doi:10.1093/treephys/tps025. 
Hereş, A.-M., Martínez-Vilalta, J., Claramunt López, B. 2012. Growth patterns in relation to drought-induced mortality at two Scots pine (Pinus sylvestris L.) sites in NE Iberian Peninsula. Trees 26:621-630.

Hobbs, R., Arico, S., Aronson, J., Baron, J.S., Bridgewater, P., Cramer, V.A., Epstein, P.R., Ewel, J.J. Klink, C.A., Lugo, A.E., Norton, D. Ojima, D., Richardson, D.M., Sanderson, E.W., Valladares, F., Vilà, M., Zamora, R., Zobel, M. 2006. Novel ecosystems: theoretical and management aspects of the new ecological world order. Global Ecology and Biogeography 15:1-7.

Kloster, S., Mahowald, N.M., Randerson, J.T., Lawrence P.J. 2012. The impacts of climate, land use, and demography on fires during the 21st century simulated by CLM-CN. Biogeosciences 9:509-525.

Linares, J.C., Camarero, J.J., Carreira, J.A. 2010a. Competition modulates the adaptation capacity of forests to climatic stress: insights from recent growth decline and death in relict stands of the Mediterranean fir Abies pinsapo. Journal of Ecology 98:592-603.

Linares, J.C., Camarero, J.J., Bowker, M.A., Ochoa, V., Carreira, J.A. 2010b. Stand-structural effects on Heterobasidion abietinum-related mortality following drought events in Abies pinsapo. Oecologia 164:1107-1119.

Linares, J.C., Pazo Sarria, R., Taïqui, L., Camarero, J.J., Ocho, V., Lechuga, V., Seco, J.I., Viñegla, B., Sangüesa, G., Gilarte, P., Merino, J., Carreira, J.A. 2012. Efectos de las tendencias climáticas y la degradación del hábitat sobre el decaimiento de los cedrales (Cedrus atlantica) del norte de Marruecos. Ecosistemas 21(3):7-14.

Lloret, F., Siscart, D., Dalmases, C. 2004. Canopy recovery after drought dieback in holm-oak Mediterranean forests of Catalonia (NE Spain). Global Change Biology 10:2092-2099.

Lloret, F. Piñol, J., Castellnou, M. 2009. Wildfires. En: Woodward, J. (ed.). The Physical Geography of the Mediterranean, pp. 541- 558. Oxford University Press, Oxford, UK.

Lloret, F., Escudero, A., Iriondo, J.M., Martínez-Vilalta, J., Valladares, F. 2012. Extreme climatic events and vegetation: the role of stabilizing processes. Global Change Biology 18:797-805.
Martínez-Vilalta, J., Aguadé, D., Banqué, M., Barba, J., Curiel Yuste, J., Galiano, L., Garcia, N., Gómez, M., Hereş, A.M., López, B.C., Lloret, F., Poyatos, R. Retana, J. Sus, O., Vayreda, J., Vilà-Cabrera, A. 2012. Las poblaciones ibéricas de pino albar ante el cambio climático: con la muerte en los talones. Ecosistemas 21(3):15-21.

Matías, L, Zamora, R., Castro, J. 2012. Sporadic rainy events are more critical than increasing of drought intensity for woody species recruitment in a Mediterranean community. Oecologia 169:833-844.

Millar, C.I., Stephenson, N.L., Scott L. 2007. Climate change and forests of the future: managing in the face of uncertainty. Ecological Applications $17: 2145-2151$

Peñuelas, J., Filella, I., Lloret, F. , Piñol, J., Rodà, F., Siscart, D. 2000. Effects of a severe drought on water and nitrogen use by Quercus ilex and Phyllirea latifolia. Biologia Plantarum 43:47-53.

Sánchez, E., Domínguez, M., Romera, R., López de la Franca, N., Gaertner, M.A., Gallardo, C., Castro, M. 2011. Regional modeling of dry spells over the Iberian Peninsula for present climate and climate change conditions. Climatic Change 107:625-634.

Sánchez-Salguero, R., Navarro-Cerrillo, R.M., Camarero, J.J., FernándezCancio, A., Swetnam, T.W., Zavala, M.A. 2012. Vulnerabilidad frente a la sequía de repoblaciones de dos especies de pinos en su límite meridional en Europa. Ecosistemas 21(3):31-40.

Sangüesa-Barreda, G., Linares, J.C., Camarero, J.J. 2012. Mistletoe effects on Scots pine decline following drought events: insights from withintree spatial patterns, growth and carbohydrates. Tree Physiologist 32:585-598.

Spittlehouse, D.L., Stewart, R.B. 2003. Adaptation to climate change in forest management. $B C$ Journal of Ecosystems and Management 4:1-11.

Vilà-Cabrera, A., Martínez-Vilalta, J., Vayreda, J., Retana, J. 2011. Structural and climatic determinants of demographic rates of Scots pine forests across the Iberian Peninsula. Ecological Applications 21:1162-1172. 